\title{
Changes in salicylic acid content and pathogenesis - related (PR2) gene expression during barley - Pyrenophora teres interaction
}

\author{
A. Al-Daoude*, M. Jawhar, E. Al-Shehadah, A. Shoaib, M. Orfi and M.I.E Arabi
}

Summary Net blotch (NB), caused by the necrotrophic fungal pathogen Pyrenophora teres f. teres, substantially reduces barley grain yield and quality worldwide. The role of salicylic acid (SA) signaling in NB resistance has been poorly documented. In this study, SA levels as well as the expression of the SA-responsive gene PR2 were monitored in infected leaves of two barley genotypes, Banteng (resistant) and WI2291 (susceptible), at different time points of infection. SA signaling was activated in bothgenotypes 24 hours post infection (hpi) as compared with non-inoculated plants. However, with or without pathogen pretreatment, $S A$ significantly increased $(P=0.001)$ in Banteng comparing with WI2291. RT-PCR analysis revealed that PR2 expression increases in the resistant and susceptible genotypes over the inoculation time points, with maximum expression ( 6.4 and 1.99 -fold, respectively) observed 6 dpi. PR2 expression was paralleled by an increase in leaf SA content as shown by the test coincidence $\left(F_{3,32}=4.74, P=0.001\right)$. Based on barley genotype resistance levels, our data strengthen the idea that SA signaling and $P R 2$ play a role in barley NB reduction.

Additional keywords: barley, Pyrenophora teres, PR2 gene expression, RT-PCR, salicylic acid

\section{Introduction}

Net blotch, caused by the fungal pathogen Pyrenophora Drechs. teres Smedeg. (anamorph: Drechslera teres [Sacc.] Shoem. f. Teres Smedeg.), is a common foliar disease of barley (Hordeum vulgare L.), a disease responsible for heavy crop losses (Liu et al., 2011; Wang et al., 2015). Various mechanisms for NB resistance and susceptibility appear to operate in barley. Pyrenophora teres activates different defense responses which are regulated through different plant signaling pathways, including plant hormones such as SA and pathogenesis-related (PR) proteins (Wang et al., 2011; Bogacki et al., 2008).

A number of studies have demonstrated that SA signaling pathways play important roles in resistance against fungal pathogens in plants (Trusov et al.,2009; Zwart et al., 2017). Therefore, discovery of SA targets and the understanding of their molecular

Department of Molecular Biology and Biotechnology, AECS, P.O.Box 6091 Damascus, Syria

*Corresponding author: scientific@aec.org.sy modes of action in physiological processes could help in the dissection of the SA signaling network, confirming its important role in plant responses to fungal diseases (Vásquez et al., 2015). After a pathogen attack SA levels often increase and lead to the induction of PR expression and the development of systemic acquired resistance and hypersensitive response. Furthermore, SA appears to regulate the delicate balance between proand after- cell death functions during hypersensitive response (Dorey et al., 1997; Alvarez, 2000).

Barley plants produce enzymes that digest fungal cell walls to stop fungal penetration. However, since all true fungi contain chitin as a primary structural component of their cell walls, the chitinase family of PR proteins is of particular importance (Wessels, 1994). Chitin in fungal cell walls can be hydrolyzed by chitinases into smaller oligomers or monomers (Bishop et al., 2002), so PR proteins such as $P R 2$ are known to play a major role during plant-pathogenic fungus interactions (Collinge et al., 1993; Dangl and Jones, 2001).

Quantitative PCR ( $q P C R$ ) is now a well- 
established method forquantifying the relative expression level of a particular transcript and determines its expression after being exposed to a specific alteration, such as pathogen infection (Kralik and Ricchi 2017). In the present work, we studied the defense responses of two barley genotypes Banteng and WI 2291, which are integrated in international breeding programs aimed at developing NB resistant barley genotypes. Banteng was described as a highly resistant to $P$. teres (Arabi et al., 2003), i.e. exhibited a lower level (compared with WI2291) of NB symptom development. We thus hypothesized that SA-triggered defenses could drive contrasted levels of resistance in Banteng and WI2291, inoculated by the same pathogen isolate. Thus, the aim of the current study was to evaluate the changes in SA content and induction of $P R 2$ gene expression in two barley cultivars with different resistance to P. teres.

\section{Materials and Methods}

\section{Plant materials and pathogen inoculation}

The German genotype Banteng has proved to be the most resistant genotype to all NB isolates available so far under field and greenhouse conditions for over fifteen years (Arabi et al., 2003). For this reason, it was chosen and used in this study. A universal susceptible control genotype (cv. WI2291) from Australia was also included in the experiments. The $P$. teres single conidium isolate (NB4) tested was the most Syrian virulent pathotype to all barley genotypes available up to now (Arabi et al., 2003). The fungus was incubated in Petri dishes containing potato dextrose agar (PDA, DIFCO, Detroit, MI, USA) for 8 days at $20-22^{\circ} \mathrm{C}$ in the dark. Conidia were collected in $10 \mathrm{~mL}$ sterile distilled water and the suspension was adjusted to $2 \times 10^{4}$ conidia/mL using hemacytometer. A surfactant (polyoxyethylene-20-sorbitan monolaurate) was added $(100 \mu \mathrm{L} / \mathrm{L})$ to the conidial suspension to facilitate dispersion of the inoculum over leaf surfaces. Pyrenophora teres inoculum preparation, inoculation, and post-inoculation were similar to those described by Abu Qamar et al. (2008). Barley plants were grown in the greenhouse and inoculated at the twoto three-leaf stage with the second leaf fully expanded.

\section{SA quantification}

Pooled samples containing the fourth leaf of 20 independent plants/genotype where used for SA quantification. Pooled samples were prepared from leaves taken 24,48 and $72 \mathrm{hpi}$, respectively. For each time case studied, six pooled sample replicates were used for quantification. SA was extracted from approximately $200 \mathrm{mg}$ of freshly ground leaves in $1.5 \mathrm{ml}$ tubes following the method described by Trapp et al. (2014), with minor modifications. Briefly, 100 mg of plant material were dried overnight in a freeze drier at $-42^{\circ} \mathrm{C}$. The extraction was achieved by adding $1.0 \mathrm{~mL}$ of ethyl acetate, dichloromethane, isopropanol, $\mathrm{MeOH}: \mathrm{H}_{2} \mathrm{O}$ into each tube containing dry plant material. Samples were shaken for $30 \mathrm{~min}$ and centrifuged at $16,000 \mathrm{~g}$ and $4^{\circ} \mathrm{C}$ for $5 \mathrm{~min}$. The supernatant was transferred into a new 1.5 micro-centrifuge tube and dried in a speed vac. After drying, $100 \mu \mathrm{L}$ of $\mathrm{MeOH}$ was added to each sample, homogenized under vortex and centrifuged at $16,000 \mathrm{~g}$ and $4^{\circ} \mathrm{C}$ for $10 \mathrm{~min}$. The supernatant was analyzed by a high-performance liquid chromatography coupled mass spectrophotometer (HPLCMS/MS) system (Agilent Technologies, Böblingen, Germany). Changes in SA content were compared to the control for each time point. Six independent repetitions were performed for each time point. Data were analyzed using the standard deviation and t-test methods.

\section{RNA isolation and cDNA synthesis}

Primary leaves from three individual biological replicates were collected at 24,48 and $72 \mathrm{hpi}$, and homogenized with a tube pestle in liquid nitrogen. mRNA was extracted with the Nucleotrap mRNA mini kit (Macherey-Nagel, MN, Germany) following the 
manufacturer's instructions. RNA was used for cDNA synthesis with the QuantiTect Reverse Transcription Kit (Qiagen) following the manufacturer's instructions and the resulting $\mathrm{CDNA}$ was stored at $-20^{\circ} \mathrm{C}$. At the same time points, samples from mock inoculated plants were collected as controls.

\section{Semi quantitative RT-PCR}

PCR primers for $P R 2$ were designed based on the CDNA sequences of barley available at NCBI (http://www.ncbi.nlm.nih. gov) database (Id:M23548.1) using Primer 3 software $\left(5^{\prime}\right.$ CAGCGAATGCTCCAATGAAGA $3^{\prime}$ and $5^{\prime}$ TACCCTGCCGTGAACATCAAG $3^{\prime}$ ). $P C R$ reactions were performed in a $50-\mu \mathrm{L}$ final volume including $1 \mu \mathrm{L}$ of ten times diluted cDNA template, $5 \mu \mathrm{L}$ of $10 \mathrm{X}$ amplification buffer (Thermo Scientific, USA), $1 \mu \mathrm{L}$ of 200 $\mu \mathrm{M}$ deoxynucleotide triphosphates (Thermo Scientific, USA), $1 \mu \mathrm{L}$ of 10 pico-molar of each primer, $0.2 \mu \mathrm{L}(1 \mathrm{U})$ of Taq DNA polymerase (MBI Fermentas, York, UK) and 40.8 $\mu \mathrm{L}$ of $\mathrm{PCR}$ grade water. $\mathrm{PCR}$ reactions were performed on a thermocycler (Biometra) with the following program: an initial denaturing step at $94^{\circ} \mathrm{C}$ for $4 \mathrm{~min}$, followed by 30 cycles of $94^{\circ} \mathrm{C}$ for $30 \mathrm{~s}, 57^{\circ} \mathrm{C}$ for $30 \mathrm{~s}, 72^{\circ} \mathrm{C}$ for 1 min with a final extension at $72^{\circ} \mathrm{C}$ for $10 \mathrm{~min}$. PCR products were separated using $1 \%$ agarose gels, stained with ethidium bromide and observed on a UV transilluminator. PCR was performed three times for each primer using the same cDNA sample in order to confirm the reproducibility of the results.

\section{qRT-PCR assay}

Quantitative real-time PCR ( $q P C R$ ) was performed using the method described by Derveaux et al. (2010). Data was checked by qRT-PCR dissociation curve analysis using stepone software (v2.3). The fluorescence readings of six replicated samples were averaged and the blank value (without DNA control) was subtracted. $P R 2$ relative expression levels were determined using the average cycle threshold (CT). Average CT values were calculated from the triplicate experiment conducted for each gene, with the
$\Delta C T$ value determined by subtracting the average $C T$ value of genes from the $C T$ value of EF1a gene. Finally, the equation $2^{-\Delta \Delta C T}$ was used to estimate $P R 2$ relative expression level (Livak and Schmittgen, 2001). Standard deviation was calculated from the replicated experimental data. The statistical analysis was conducted through the Tukey's test at the 0.05 level. The assumption of coincidence was tested using the ANOVA procedure implemented in the software package Statistica 6.1.

\section{Results and Discussion}

In this study, we used two barley genotypes with different resistance to $P$. teres infection. As shown in Figure 1, P. teres produced netlike striated lesions surrounded by chlorosis or necrosis, and these symptoms were more severe on the susceptible genotype 'WI2291' after 10 days of infection. These results are in agreement with our previous observations under natural field conditions (Arabi et al., 2003).

Further studies of barley- $P$. teres interactions by measuring changes in the leaf SA content and $P R 2$ gene expression at four early time points after pathogen challenge, showed that SA levels of infected barley leaves increased $24 \mathrm{hpi}$ in comparison with non-inoculated plants (Fig. 2). With or without pathogen pretreatment, the tolerant genotype Banteng contained three-fold or greater total SA than the susceptible genotype WI2291 (24hpi). It was found that Banteng contained significantly $(\mathrm{P}=0.001)$ higher levels of total SA thanWI2291 a teach time point investigated (Fig. 2), which might reflect the expected role of SA in signaling events during $P$. teres infection. This result could support the findings published by Häffner et al. (2014), stating that the endogenous SA level in a plant is the main cause of susceptibility versus resistance in barley, since pathogen infection may induce plant responses regulated by SA. In addition, SA accumulation has been widely used as a reliable marker of elevated defense responses 
(a)

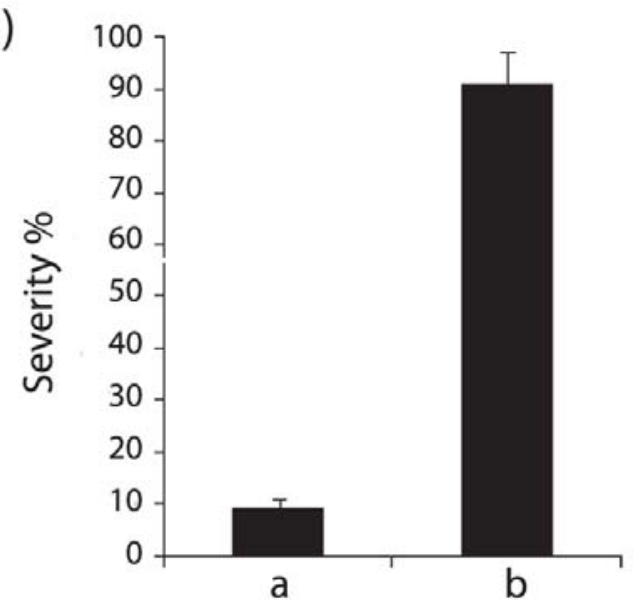

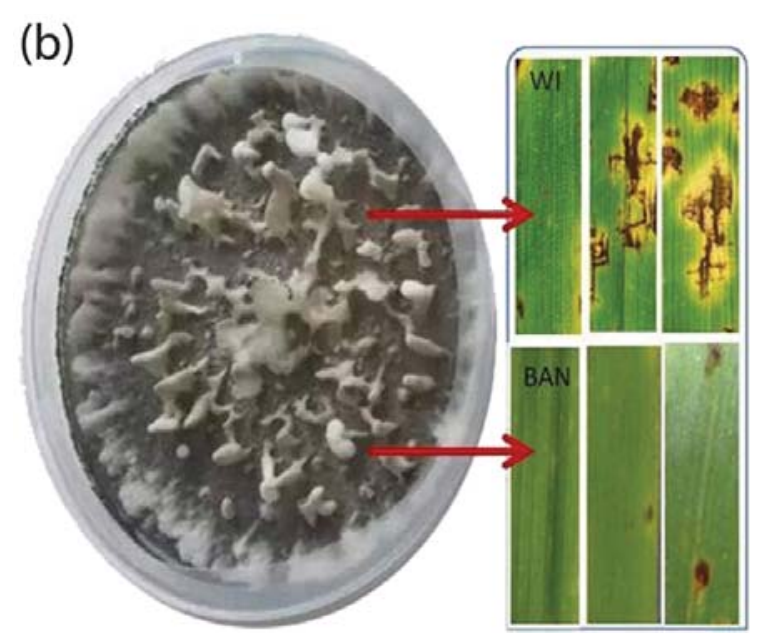

Figure 1. a) Frequency of disease reactions incited on barley (a) resistant cv. Banteng and (b) susceptible cv. WI2291, 10 days after Pyrenophora teres infection. b) Disease symptoms on the resistant (BAN) and susceptible (WI) barley genotypes, which were measured using the scale described by Abu Qamar et al. (2008).

(a)

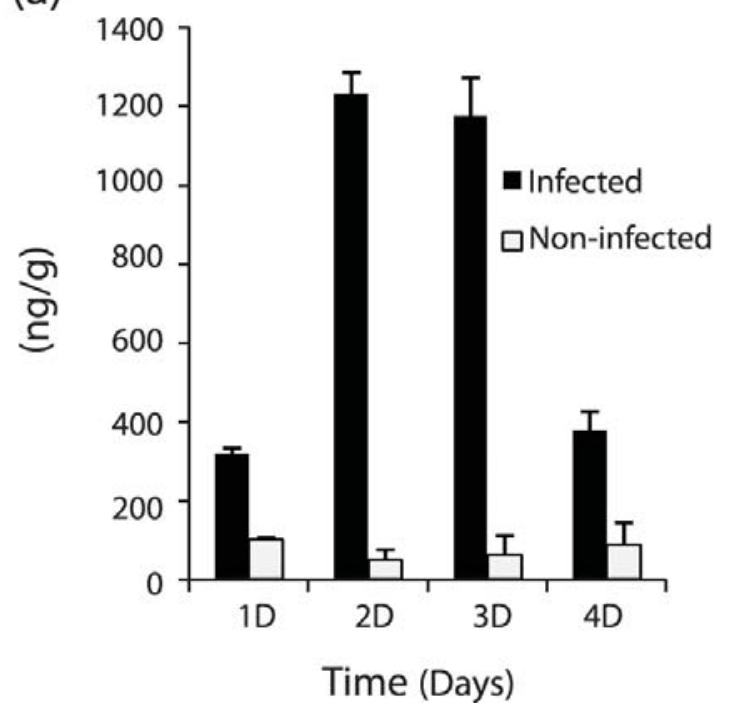

(b)

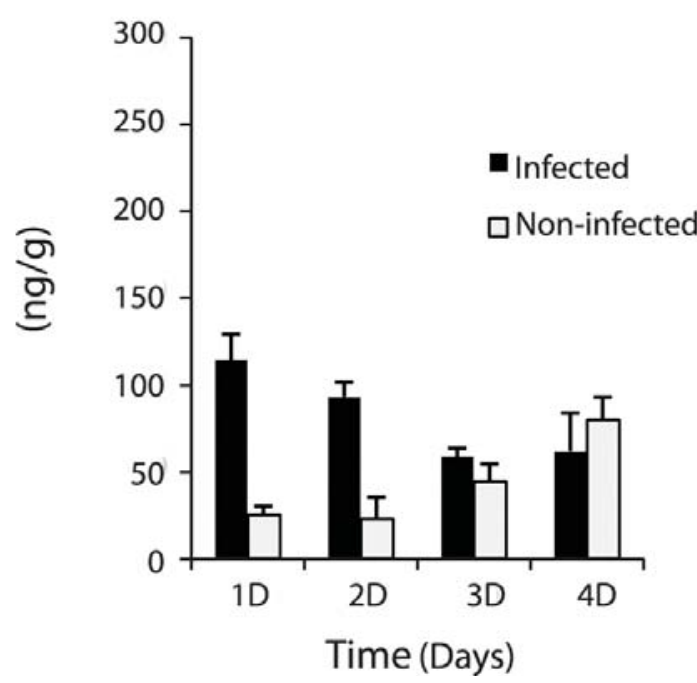

Figure 2. Quantification of total salycilic acid in barley leaves 1, 2,3 and 4 days post inoculation with Pyrenophora teres in (a) the resistant cv. Banteng and (b) the suscepitible cv. WI2291. Error bars represent the stantard error of the means ( $n=$ 3).

and is closely associated with redox homeostasis, hypersensitive response, or systemic acquired resistance (Alvarez, 2000; Dong, 2004).

Semi quantitative RT-PCR analysis demonstrated that attack of barley by $P$. teres induced $P R 2$ accumulation in infected plants as compared with the un-infected controls and it was inversely regulated $24 \mathrm{~h}$ post inoc- ulation i.e, it was repressed in the susceptible cultivar WI2291 while being induced in the tolerant genotype Banteng (Fig. 3). Moreover, RT-PCR expression analysis revealed that the $P R 2$ expression increased in the resistant and susceptible genotypes over the inoculation time points, with the highest expression (6.4 and 1.99-fold for Banteng and WI2291, respectively) observed at $6 \mathrm{dpi}$. PR2 


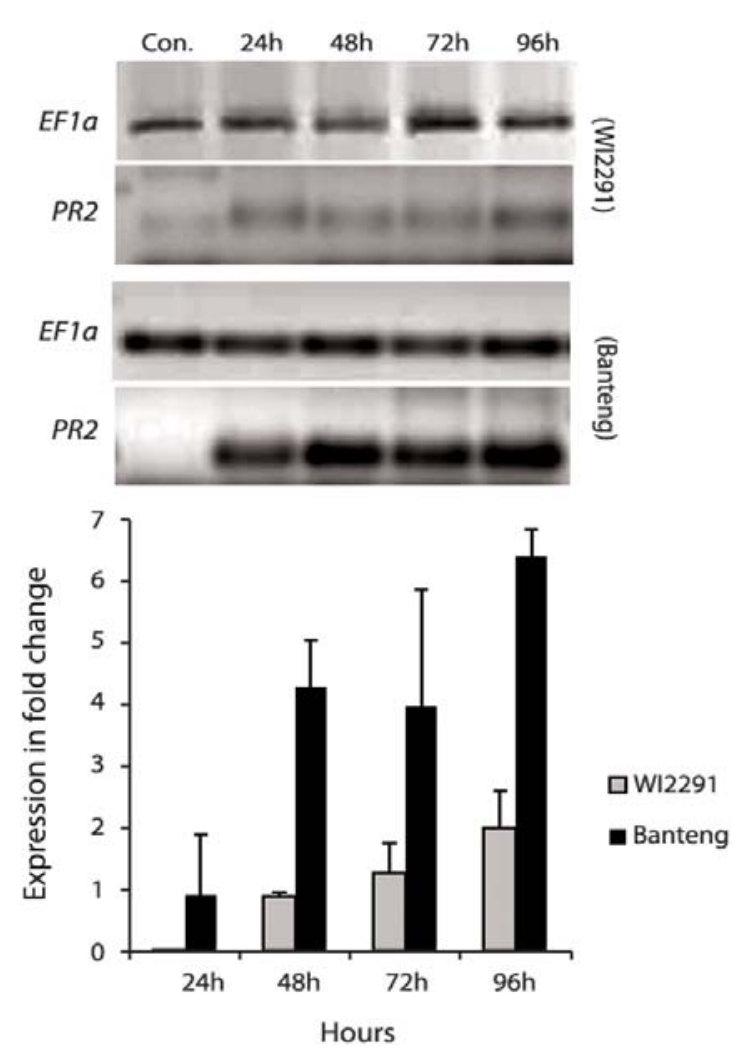

Figure 3. Relative expression profiles of $P R 2$ gene in the resistant cv. Banteng and in the susceptible cv. WI2291, 24, 48, 72 and 96 hours after infection by Pyrenophora teres.

encodes for a 1,3-ß-glucanase (Simmons, 1994), belonging to the glycoside hydrolases family (Opassiri et al., 2010). 1, 3-ß-glucanase hydrolyses the $B-O$-glycosidic bond of B-glucan in plant cell walls, resulting in cell wall loosening and expansion (Akiyama et al., 2009). This phenomenon may be the cause of barley cell wall leakage during $N B$ infestations.

Our data showed that $P R 2$ gene exhibited a differential expression $(P=0.01)$ in the tolerant and susceptible barley genotypes and was closely related to the increase of the SA level. The SA marker PR2 was upregulated 3-fold in infected leaves of the tolerant Banteng than in the susceptible WI2291 (Fig. 2). PR2 expression was paralleled by an increase in leaf salicylic acid (SA) content as shown by the coincidence test $\left(F_{3,32}=4.74, P\right.$ $=0.001)$. This is supported by previous works indicating that $S A$ is involved in the regulation of induced immunity in barley through the induction of PR proteins with chitinase, $\beta-1,3$-glucanase and peroxidase enzyme activity, both locally and systemically (Bindschedler et al., 1998).

Our data show that the contribution of the SA pathway to the resistance response appears to depend on the plant genotype. The NB tolerant genotype Banteng used for this study was proved to be the most resistant genotype to all $P$. teres isolates available so far. The higher activities of the selected defense genes such as PR2 and higher level of SA in infected Banteng leaf tissues compared with the susceptible genotype WI 2291 may explain its high level of resistance.

This study provides information about the role of SA in resistance of barley against the necrotrophic foliar pathogen $P$. teres. In addition, it highlights that SA may increase in response to $P$. teres infection in different barley genotypes. It is also noteworthy that $P R 2$ has a higher constitutive expression and faster induction in the tolerant genotype as compared with the susceptible one. Our results suggested that not only SA is important for the induction of defense-like responses but, in the absence of pathogen attack, SA may sustain basal expression levels of genes associated with resistance responses and may keep the defense system primed.

The authors thank the Director General of AECS and the Head of Biotechnology Department for their help throughout the period of this research.

\section{Literature cited}

Abu Qamar, M., Liu, Z. H., Faris, J. D., Chao, S., Edwards, M. C., Lai, Z., Franckowiak, J.D. and Friesen, T.L. 2008. A region of barley chromosome $6 \mathrm{H}$ harbors multiple major genes associated with the net type net blotch resistance. Theoretical and Applied Genetics, 117: 1261-1270.

Akiyama, T., Jin, S., Yoshida, M., Hoshino, T., Opassiri, R. and Cairns, J.R.K. 2009. Expression of an endo-(1,3;1,4)-beta-glucanase in response to wounding, methyl jasmonate, abscisic acid and ethephon in rice seedlings. Journal of Plant Physiology, 166: 1814-1825. 
Alvarez, M.E. 2000. Salicylic acid in the machinery of hypersensitive cell death and disease resistance. Plant Molecular Biology, 44: 429-442.

Arabi, M.I.E., Al-safadi, B. and Charbaji, T. 2003. Pathogenic Variation among Isolates of Pyrenophora teres the Causal Agent of Barley Net Blotch. Journal of Phytopathology, 151: 376-382.

Bindschedler, V.L., Métraux, J.P. and Schweizer, P. 1998. Salicylic acid accumulation in barley is pathogen specific but not required for defensegene activation. Molecular Plant-Microbe Interactions, 11: 702-705.

Bishop, J.G, Dean, A.M. and Mitchell-Olds, T. 2002. Rapid evolution in plant chitinases: molecular targets of selection in plant-pathogen coevolution. Proceedings of the National Academy of Sciences of the United States of America, 97: 53225327.

Bogacki, P., Oldach, K. H., and Williams, K.J. 2008. Expression profiling and mapping of defense response genes associated with the barleyPyrenophora teres incompatible interaction. Molecular Plant Pathology, 9: 645-660.

Collinge, D.B., Kragh, K.M., Mikkelsen, J.D., Nielsen, K.K., Rasmussen, J., et al. 1993 Plant chitinases. The Plant Journal, 3: 31-40.

Dangl, J.L. and Jones, J.D.G. 2001. Plant pathogens and integrated defense responses to infection. Nature, 411: 826-833.

Derveaux, S., Vandesompele, J. and Hellemans, J., 2010. How to do successful gene expression analysis using real-time PCR. Methods, 50: 227230.

Dong, X.N., 2004. NPR1 all things considered. Current Opinion in Plant Biology, 7: 547-552.

Häffner, E., Karlovsky, P., Splivallo, R., Traczewska, A. and Diederichsen, E. 2014. ERECTA, salicylic acid, abscisic acid, and jasmonic acid modulate quantitative disease resistance of Arabidopsis thaliana to Verticillium longisporum. BMC Plant Biology, 14: 71-85.

Kralik, P. and Ricchi, M. 2017. A Basic Guide to Real Time PCR in Microbial Diagnostics: Definitions, Parameters, and Everything. Frontiers in Microbiology, 8: 108.

Livak, K.J. and Schmittgen, T.D. 2001. Analysis of relative gene expression data using real-time quantitative PCR and the 2(-Delta Delta $C(T)$ ) Method. Methods, 25: 402-408.
Liu, Z., Ellwood, S.R., Oliver, R.P. and Friesen, T.L., 2011. Pyrenophora teres profile of an increasingly damaging barley pathogen. Molecular Plant Pathology, 12: 1-19.

Opassiri, R., Maneesan, J., Akiyama, T., Pomthong, S., Jin B., Kimura, A. and Ketudat Cairns, J.R. 2010. Rice Os4BGlu12 is a wound-induced $\beta$-glucosidase that hydrolyzes cell wall- $\beta$ glucan-derived oligosaccharides and glycosides. Plant Science, 179: 273-278.

Simmons, C.R., 1994. The physiology and molecular biology of plant 1,3-P-o-glucanases and 1,3;1,4P-o-glucanases. Critical Review in Plant Sciences, 13: 325-387

Trapp, M.A., De Souza, G.D., Filho, E.R., Boland, W. and Mithöfer, A. 2014. Validated method for phytohormone quantification in plants. Frontiers in Plant Science, 5: 417.

Trusov, Y., Sewelam, N., Rookes, J.E., Kunkel, M., Nowak, E., Schenk, P.M. and Botella, J.R. 2009. Heterotrimeric $\mathrm{G}$ proteins-mediated resistance to necrotrophic pathogens includes mechanisms independent of salicylic acid-, jasmonic acid/ethylene- and abscisic acid-mediated defense signaling. The Plant Journal, 58: 69-81.

Vásquez, A.H., Salinas, P. and Holuigue, L. 2015. Salicylic acid and reactive oxygen species interplay in the transcriptional control of defense genes expression. Frontiers in Plant Science, 6: 171.

Wang, X., Mace, E.S., Platz, G.J., Hunt, C.H., Hickey, L.T., Franckowiak, J.D. and Jordan, D.R. 2015. Spot form of net blotch resistance in barley is under complex genetic control. Theoretical and Applied Genetics, 108: 1064-1070.

Wessels, J.G.H. 1994. Developmental regulation of fungal cell-wall formation. Annual Review of Phytopathology, 32: 413-437.

Zwart, L., Berger, D.K., Moleleki, L.N., van der Merwe, N.A., Myburg, A.A. and Naidoo, S. 2017. Evidence for salicylic acid signalling and histological changes in the defence response of Eucalyptus grandis to Chrysoporthe austroafricana. Scientific Reports, 7:45402. doi:10.1038/srep45402.

Received: 17 May 2018; Accepted: 9 July 2018 


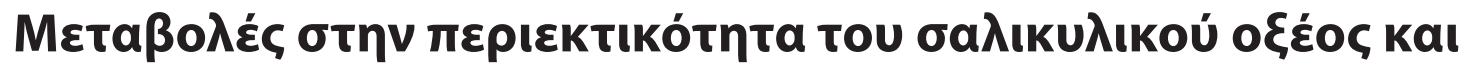

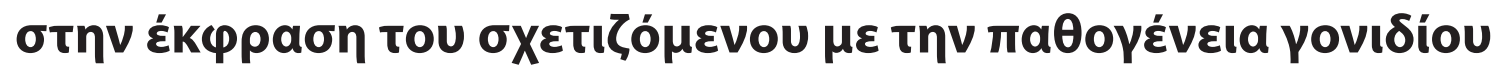

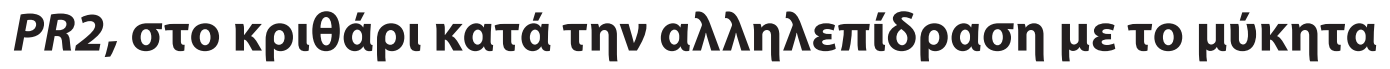 Pyrenophora teres
}

\author{
A. Al-Daoude, M. Jawhar, E. Al-Shehadah, A. Shoaib, M. Orfi and M.I.E Arabi
}

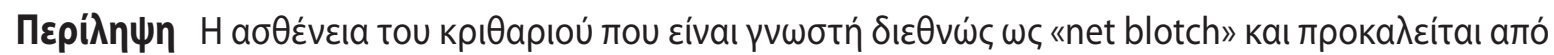

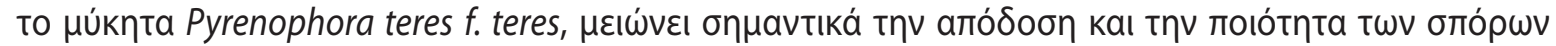

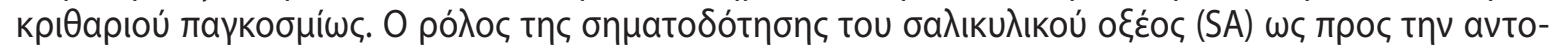

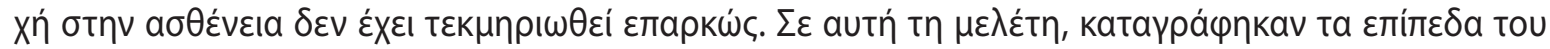

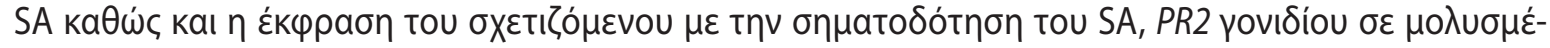

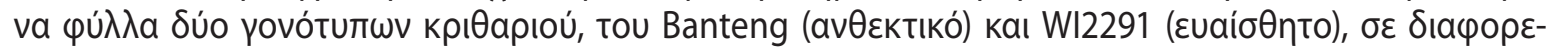

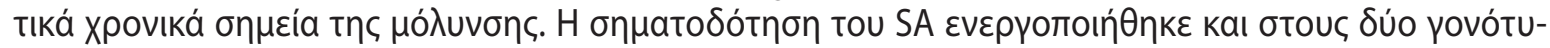

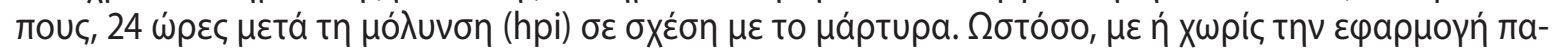

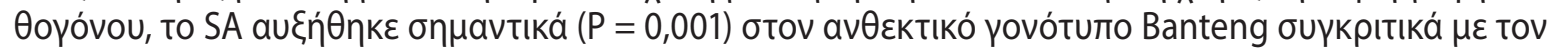

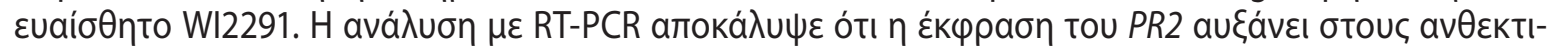

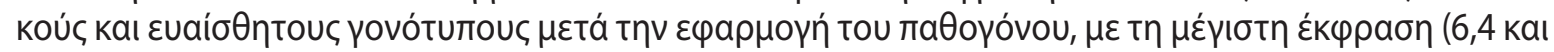

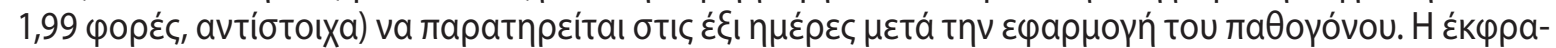

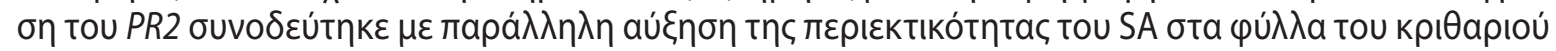

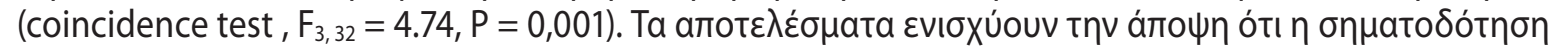

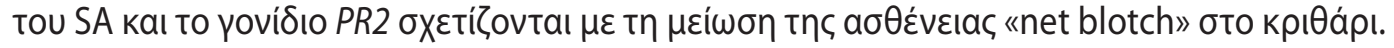

